Onkologe 2013 · 19:262-264

DOI 10.1007/s00761-012-2405-9

Online publiziert: 17. März 2013

(c) Springer-Verlag Berlin Heidelberg 2013

\section{A. Adamietz ${ }^{1}$ P. M. Schlag ${ }^{2}$}

${ }^{1}$ Klinik für Strahlentherapie und Radio-Onkologie, Marienhospital Herne,

Klinikum der Ruhr-Universität Bochum, Herne

${ }^{2}$ Charité Comprehensive Cancer Center, Charité - Universitätsmedizin Berlin, Campus Mitte, Berlin
Inzwischen können moderne onkologische Therapieverfahren Prognose und Überleben bei vielen Tumorerkrankungen deutlich verbessern. Insbesondere bei potenziell kurativen behandelbaren Stadien stehen intensive und komplexe Therapieformen, die eine komplette Elimination vitaler Tumorzellen aus dem Körper zum Ziel haben, im Vordergrund. Die Effektivität dieser Verfahren wird allerdings durch eine Reihe von Nebenwirkungen erkauft. Unterschiedliche supportive Maßnahmen können diese lindern. Kann ein Tumor nicht vollständig beseitigt werden, ist meist eine nebenwirkungsreiche und belastende Behandlung wenig sinnvoll. Parallel müssen dann jedoch meist die symptomatischen Folgen der Tumorprogredienz mitbehandelt werden. In der Phase der malignen Erkrankung, in der keine gegen den Tumor unmittelbar gerichteten Maßnahmen mehr sinnvoll sind, werden die Patienten situativ zur Besserung von Funktionsstörungen und der allgemeinen Befindlichkeit behandelt.

Insgesamt kann die kurative wie palliative Tumortherapie durch supportive Maßnahmen effektiv flankiert werden, um Durchführbarkeit und Erträglichkeit der Therapie zu optimieren sowie Nebenwirkungen der Behandlung oder Auswirkungen des Tumorwachstums zu lindern. Die European Organisation for Research and Treatment of Cancer (EORTC) definiert die Best Supportive Care als:

Behandlungsmaßnahme für Krebspatienten, welche das multiprofessionelle Bemühen um die individuellen physischen, psy- chosozialen, spirituellen und kulturellen Bedürfnisse umfassen und welche zu jedem Zeitpunkt der Krankheit für Patienten allen Alters und unabhängig von der gegenwärtigen Behandlungsintention alle gegen die Krankheit gerichteten Maßnahmen mit einschließt.

Diese Definition verdeutlicht, dass damit die Palette der supportiven Verfahren sehr breit gefächert sein muss. Sie impliziert auch die Notwendigkeit der transdisziplinären Kommunikation und Zusammenarbeit.

\section{》) Die Palette der \\ supportiven Verfahren muss \\ sehr breit gefächert sein}

Aufgrund dieser Vielfalt supportiver Therapiemaßnahmen ist es unmöglich, zeitgleich ihr gesamtes Spektrum in einem Schwerpunktheft darzustellen. Deshalb wurden für diese Ausgabe von Der Onkologe einige aktuelle Themen ausgewählt. Sie beschäftigen sich mit der Rolle des Chirurgen in der symptomorientierten Supportivtherapie, setzen sich mit praktischen Anwendungen der Supportivmaßnahmen in der Strahlentherapie auseinander, erkunden die Möglichkeiten der Prophylaxe und Therapie der Fatigue, schildern die Bedeutung onkoneuronaler Antikörper in der Pathogenese unterschiedlicher paraneoplastischer neurologischer Syndrome (PNS) und deren Bedeutung für die weitere Abklärung, Klassifikation und Therapie dieser Syndrome.
Im Beitrag von Bork et al. wird die Rolle der symptomorientierten Supportivmaßnahmen aus chirurgischer Sicht detailliert beleuchtet. Das vonseiten der Chirurgie vorgehaltene Spektrum palliativmedizinischer Maßnahmen ist groß. Es reicht und bezieht sich auf alle Körperregionen. Es umfasst Entlastungsverfahren, Teilresektionen und Funktionswiederherstellung und ist damit häufig in der Lage, die Symptome einer Tumorerkrankung nachhaltig zu lindern. Die Kenntnis dieser Möglichkeiten erleichtert dem betreuenden Arzt unter Berücksichtigung der besonderen individuellen Situation, die beste Therapie für seinen Patienten auszuwählen. Der Chirurg ist dabei sowohl präventiv symptomorientiert tätig als auch in akut lebensbedrohlichen Situationen aktiv. Die Aufgaben und Fragestellungen sind auch hierbei - von der Beseitigung tumorbedingter Passagestörungen, Kompressionszuständen oder einer Tumorperforation bis zur Unterbindung einer akuten Tumorblutung - breit gestreut.

Die Strahlentherapie ist zwar auch eine lokoregionale Maßnahme in der Tumorbehandlung, kann aber symptomatische Auswirkungen auf den gesamten Körper haben. Sie erfordert sowohl auf das bestrahlte Organ bezogene, lokal wirksame als auch breit gefächerte allgemeine Supportivmaßnahmen. Im Beitrag von Riesenbeck et al. zur Behandlung der Nebenwirkungen der Strahlentherapie werden Ursachen, Klinik und Möglichkeiten der supportiven Therapie geschildert. Auch hier steht die Kontrolle der Symptome im Vordergrund. Aber auch die praktischen 
Hier steht eine Anzeige.

黛 Springer 
Aspekte der Therapie im Alltag, insbesondere im Rahmen unterschiedlicher Therapiestrategien wie präoperativer, postoperativer, definitiver und palliativer Bestrahlung und die Symptombekämpfung in diesen verschiedenen therapeutischen Konstellationen werden erläutert.

Eine Erschöpfung im Zusammenhang mit der Tumorerkrankung tritt bei nahe$\mathrm{zu}$ allen Patienten therapieassoziiert und bei etwa einem Drittel der Patienten (je nach Entität) auch Jahre nach erfolgreicher Therapie auf. Fatigue hat eine erhebliche Auswirkung auf die Lebensqualität und auch auf die Behandlungsadhärenz und den Therapieverlauf. Der Beitrag von Rüffer et al. zur Fatigue setzt sich detailliert mit der facettenreichen Thematik auseinander, schildert die Prinzipien der Diagnostik und Therapie und beschäftigt sich mit den Möglichkeiten der Prophylaxe dieses sehr verbreiteten und lästigen Syndroms.

Die parakrine Aktivität der Tumorzellen kann zu einer Reihe von komplexen Symptomen führen, die (teilweise in Abhängigkeit von der Entität) alle Organsysteme des Körpers betreffen können. Paraneoplastische neurologische Syndrome (PNS) im Rahmen einer Tumorerkrankung gehören zu den seltenen neurologischen Erkrankungen. Ihre Natur und Ätiologie blieben lange Jahre im Dunkeln. Mit der Entdeckung onkoneuronaler Antikörper und einer europaweiten Erfassung von PNS wurde ein tiefgreifendes Verständnis dieser tumorassoziierten Erkrankungen gewonnen. Der Beitrag von Lindeck-Pozza zu den neurologischen Syndromen paraneoplastischer Natur erläutert die Zusammenhänge zwischen kürzlich beschriebenen onkoneuronalen Antikörpern und unterschiedlichen Formen der paraneoplastischen Syndrome des zentralen oder peripheren Nervensystems.

> Die supportive Therapie bei Tumorerkrankungen wird umso komplexer, je fortgeschrittener das Tumorleiden ist.

Sie erfordert einen sehr hohen Grad der Professionalisierung und Individualisierung. Kommunikation, Interdisziplinarität und Qualitätssicherung spielen damit eine wichtige Rolle. Wie im Beitrag von Kloke aufgezeigt und auch aufgrund eines großen persönlichen Engagements konnten durch internationalen Informationsaustausch nationale Programme zur Qualitätssicherung in der Palliativmedizin erfolgreich gestartet und ausgebaut werden. Auch die finanzielle Absicherung der symptomatischen Supportivtherapie der Tumorpatienten wurde im Rahmen der Komplexpauschalen auf die Beine gestellt.

Auch wenn diese Ergebnisse zwar einen wichtigen weiteren Fortschritt in der Behandlung von Tumorpatienten bedeuteten, sind wir noch weit von einer flächendeckenden, ausreichenden supportiven und palliativen Versorgung entfernt. Die Diskrepanzen zwischen den begründeten Erwartungen, Möglichkeiten und Anforderungen und der täglichen Umsetzung gilt es weiter abzubauen. Dieses spezielle Wissen und damit auch die Ziele der Supportivtherapie generell weiter zu vertiefen, ist Anliegen dieses Schwerpunkthefts.

\section{A. Arlanamata}

Prof. Dr. I. Adamietz

Für die Herausgeber des Schwerpunktthemas

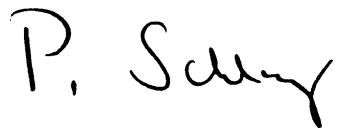

Prof. Dr. Dr. h.c. P. M. Schlag Für die Herausgeber

\section{Korrespondenzadresse}

\section{Prof. Dr. I. A. Adamietz}

Klinik für Strahlentherapie und

Radio-Onkologie, Marienhospital Herne,

Klinikum der Ruhr-Universität Bochum

Hölkeskampring 40, 44625 Herne

irenaeus-anton.adamietz@

marienhospital-herne.de

\section{Oncoflow}

Innovative Software unterstützt interdisziplinäres Tumorboard

Krebsbehandlungen im Kopf-Hals-Bereich besser zu steuern ist das Ziel von HNO-

Ärzten in Leipzig. Mit der neu entwickelten Software Oncoflow arbeiten sie daher nun im computergestützten Tumorboard. Das neue Programm bündelt alle patientenbezogenen Daten und stellt sie auf einer Benutzeroberfläche zur Verfügung. Neben Laborbefunden, Untersuchungsergebnissen und Arztberichten fließen hier auch alle Bilddaten aus Röntgen-, MRT- und CT-Untersuchungen ein. Diese werden mit allen anderen Daten zu dreidimensionalen Modellen des Tumors zusammengeführt und auf einem Bildschirm dargestellt. „Diese neue Plattform ist für uns ein ungeheuer wichtiges Werkzeug - anhand der Daten und Auswertungen können wir uns in unseren interdisziplinären Tumorboardbesprechungen ein sehr umfassendes Bild davon verschaffen, mit welchem Tumor wir es zu tun haben und welche Maßnahmen die besten sind", erläuterte Andreas Dietz, Direktor der HNO-Klinik am Universitätsklinikum Leipzig. Für die Patienten bedeute dies, dass die Ärzte die Behandlung noch besser an den konkreten Fall anpassen könnten.

Quelle: Deutsches Ärzteblatt, www.aerzteblatt.de 